\title{
Model Predictive Control based on Finite Impulse Response Models
}

\author{
Prasath, Guru; Jørgensen, John Bagterp
}

Published in:

American Control Conference 2008

Link to article, DOI:

10.1109/ACC.2008.4586531

Publication date:

2008

Document Version

Publisher's PDF, also known as Version of record

Link back to DTU Orbit

Citation (APA):

Prasath, G., \& Jørgensen, J. B. (2008). Model Predictive Control based on Finite Impulse Response Models. In American Control Conference 2008 IEEE. https://doi.org/10.1109/ACC.2008.4586531

\section{General rights}

Copyright and moral rights for the publications made accessible in the public portal are retained by the authors and/or other copyright owners and it is a condition of accessing publications that users recognise and abide by the legal requirements associated with these rights.

- Users may download and print one copy of any publication from the public portal for the purpose of private study or research.

- You may not further distribute the material or use it for any profit-making activity or commercial gain

- You may freely distribute the URL identifying the publication in the public portal

If you believe that this document breaches copyright please contact us providing details, and we will remove access to the work immediately and investigate your claim 


\title{
Model Predictive Control based on Finite Impulse Response Models
}

\author{
Guru Prasath and John Bagterp Jørgensen* \\ Informatics and Mathematical Modelling \\ Technical University of Denmark \\ DK-2800 Kgs. Lyngby, Denmark \\ $\{$ guru, jbj\}@imm.dtu.dk
}

\begin{abstract}
We develop a regularized $l_{2}$ finite impulse response (FIR) predictive controller with input and input-rate constraints. Feedback is based on a simple constant output disturbance filter. The performance of the predictive controller in the face of plant-model mismatch is investigated by simulations and related to the uncertainty of the impulse response coefficients. The simulations can be used to benchmark $l_{2}$ MPC against FIR based robust MPC as well as to estimate the maximum performance improvements by robust MPC.
\end{abstract}

\section{INTRODUCTION}

We investigate the effect of uncertain models on the performance of a regularized $l_{2}$ model predictive controller with input and input-rate constraints [1]. We use a finite impulse response (FIR) model for prediction of the process outputs. In contrast to state space parameterizations, the FIR model is in a form that can easily be applied in robust predictive control, i.e. predictive control based on robust linear programming [2] or second-order cone programming [3], [4]. To facilitate comparative performance studies of $l_{2}$ and robust MPC, we establish a FIR based $l_{2}$-MPC benchmark. This serves to bound the achievable performance limits of FIR based robust MPC.

This paper is organized as follows. We derive the predictive controller consisting of a regulator and an estimator in Section II. By simulation Section III illustrates the effect of uncertain impulse responses on the closed-loop MPC performance. Section IV provides closed-loop simulations with stochastic process and measurement noise. Conclusions are given in Section V.

\section{FIR MODEL BASED MPC}

Model predictive control systems consists of an estimator and a regulator as illustrated in Figure 1. The inputs to the MPC are the target values, $r$, for the process outputs, $z$, and the measured process outputs, $y$. The output from the MPC is the manipulated variables, $u$.

\section{A. Plant and Sensors}

The plant is assumed to be a linear state space system

$$
\begin{aligned}
\boldsymbol{x}_{k+1} & =A \boldsymbol{x}_{k}+B u_{k}+B_{d} d_{k}+G \boldsymbol{w}_{k} \\
\boldsymbol{z}_{k} & =C \boldsymbol{x}_{k}
\end{aligned}
$$

with $x$ being the states, $u$ being the manipulated variables (MVs), $d$ being unmeasured disturbances, and $w$ being

\footnotetext{
* Corresponding author
}

stochastic process noise. $z$ denotes the controlled variables $(\mathrm{CVs})$. The measured outputs, $y$, are the controlled outputs, $z$, corrupted by measurement noise, $v$. Consequently

$$
\boldsymbol{y}_{k}=\boldsymbol{z}_{k}+\boldsymbol{v}_{k}
$$

The initial state, the process noise, and the measurement noise are assumed to be normally distributed stochastic vectors

$$
\begin{aligned}
\boldsymbol{x}_{0} & \sim N\left(\bar{x}_{0}, P_{0}\right) \\
\boldsymbol{w}_{k} & \sim N_{i i d}(0, Q) \\
\boldsymbol{v}_{k} & \sim N_{i i d}(0, R)
\end{aligned}
$$

The measured output, $y$, is the signal available for feedback and used by the estimator. $u$ is the signal generated by the control system and implemented on the plant.

\section{B. Regulator}

Stable processes can be represented by the finite impulse response (FIR) model

$$
z_{k}=b_{k}+\sum_{i=1}^{n} H_{i} u_{k-i}
$$

in which $\left\{H_{i}\right\}_{i=1}^{n}$ are the impulse response coefficients (Markov parameters). $b_{k}$ is a bias term generated by the estimator. $b_{k}$ accounts for discrepancies between the predicted output and the actual output. In this paper, the output predictions used by the regulator are based on the FIR model (4). Consequently, using the FIR model (4), the regularized

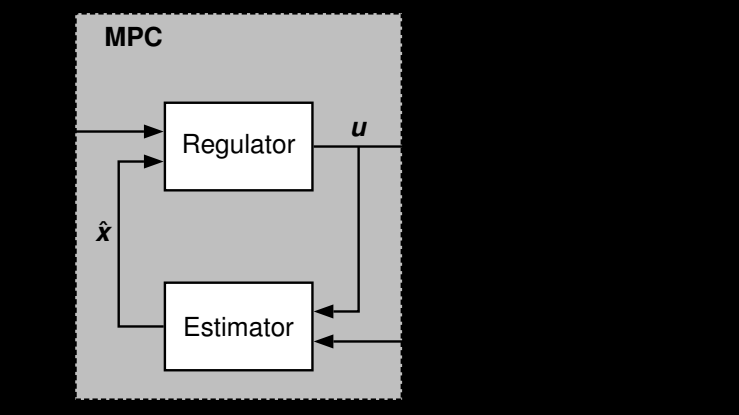

Fig. 1. Generic model predictive control system. 
$l_{2}$ output tracking problem with input constraints may be formulated as

$$
\begin{aligned}
& \min _{\{z, u\}} \phi=\frac{1}{2} \sum_{k=0}^{N-1}\left\|z_{k+1}-r_{k+1}\right\|_{Q_{z}}^{2}+\left\|\Delta u_{k}\right\|_{S}^{2} \\
& \text { s.t. } \quad z_{k}=b_{k}+\sum_{i=1}^{n} H_{i} u_{k-i} \quad k=1, \ldots, N \\
& u_{\min } \leq u_{k} \leq u_{\max } \quad k=0, \ldots, N-1 \\
& \Delta u_{\min } \leq \Delta u_{k} \leq \Delta u_{\max } k=0, \ldots, N-1
\end{aligned}
$$

in which $\Delta u_{k}=u_{k}-u_{k-1}$. In this formulation, the control and the prediction horizon are identical. If desired, a prediction horizon longer than the control horizon could be included in the formulation. However, we prefer instead to select the control horizon sufficiently long such that any boundary effects at the end of the horizon has no influence on the solution in the beginning of the horizon. (5) can be converted to a constrained linear-quadratic optimal control problem. Efficient algorithms exists for the solution of such problems with long prediction horizons, $N$. In this paper we adopt another approach and formulate a dense quadratic program in standard form that is equivalent with (5).

Define the vectors $Z, R$, and $U$ as

$$
Z=\left[\begin{array}{c}
z_{1} \\
z_{2} \\
\vdots \\
z_{N}
\end{array}\right] \quad R=\left[\begin{array}{c}
r_{1} \\
r_{2} \\
\vdots \\
r_{N}
\end{array}\right] \quad U=\left[\begin{array}{c}
u_{0} \\
u_{1} \\
\vdots \\
u_{N-1}
\end{array}\right]
$$

Then the predictions by the impulse response model $(5 \mathrm{~b})$ may be expressed as

$$
Z=c+\Gamma U
$$

For the case $N=6$ and $n=3, \Gamma$ is assembled as

$$
\Gamma=\left[\begin{array}{cccccc}
H_{1} & 0 & 0 & 0 & 0 & 0 \\
H_{2} & H_{1} & 0 & 0 & 0 & 0 \\
H_{3} & H_{2} & H_{1} & 0 & 0 & 0 \\
0 & H_{3} & H_{2} & H_{1} & 0 & 0 \\
0 & 0 & H_{3} & H_{2} & H_{1} & 0 \\
0 & 0 & 0 & H_{3} & H_{2} & H_{1}
\end{array}\right]
$$

and $c$ is

$$
c=\left[\begin{array}{c}
c_{1} \\
c_{2} \\
c_{3} \\
c_{4} \\
c_{5} \\
c_{6}
\end{array}\right]=\left[\begin{array}{c}
b_{1}+\left(H_{2} u_{-1}+H_{3} u_{-2}\right) \\
b_{2}+\left(H_{3} u_{-1}\right) \\
b_{3} \\
b_{4} \\
b_{5} \\
b_{6}
\end{array}\right]
$$

Similarly, for the case $N=6$, define the matrices $\Lambda$ and $I_{0}$ by

$$
\Lambda=\left[\begin{array}{cccccc}
I & 0 & 0 & 0 & 0 & 0 \\
-I & I & 0 & 0 & 0 & 0 \\
0 & -I & I & 0 & 0 & 0 \\
0 & 0 & -I & I & 0 & 0 \\
0 & 0 & 0 & -I & I & 0 \\
0 & 0 & 0 & 0 & -I & I
\end{array}\right] \quad I_{0}=\left[\begin{array}{c}
I \\
0 \\
0 \\
0 \\
0 \\
0
\end{array}\right]
$$

and

$$
\mathcal{Q}_{z}=\left[\begin{array}{cccc}
Q_{z} & & & \\
& Q_{z} & & \\
& & \ddots & \\
& & & Q_{z}
\end{array}\right] \mathcal{S}=\left[\begin{array}{llll}
S & & & \\
& S & & \\
& & \ddots & \\
& & S
\end{array}\right]
$$

Then the objective function (5a) may be expressed as

$$
\begin{aligned}
\phi= & \frac{1}{2} \sum_{k=0}^{N-1}\left\|z_{k+1}-r_{k+1}\right\|_{Q_{z}}^{2}+\left\|\Delta u_{k}\right\|_{S}^{2} \\
= & \frac{1}{2}\|Z-R\|_{\mathcal{Q}_{z}}^{2}+\frac{1}{2}\left\|\Lambda U-I_{0} u_{-1}\right\|_{\mathcal{S}}^{2} \\
= & \frac{1}{2}\|c+\Gamma U-R\|_{\mathcal{Q}_{z}}^{2}+\frac{1}{2}\left\|\Lambda U-I_{0} u_{-1}\right\|_{\mathcal{S}}^{2} \\
= & \frac{1}{2} U^{\prime}\left(\Gamma^{\prime} \mathcal{Q}_{z} \Gamma+\Lambda^{\prime} \mathcal{S} \Lambda\right) U \\
& \quad+\left(\Gamma^{\prime} \mathcal{Q}_{z}(c-R)-\Lambda^{\prime} \mathcal{S} I_{0} u_{-1}\right)^{\prime} U \\
& \quad+\left(\frac{1}{2}\|c-R\|_{\mathcal{Q}_{z}}^{2}+\frac{1}{2}\left\|I_{0} u_{-1}\right\|_{\mathcal{S}}^{2}\right) \\
= & \frac{1}{2} U^{\prime} H U+g^{\prime} U+\rho
\end{aligned}
$$

in which

$$
\begin{aligned}
H & =\Gamma^{\prime} \mathcal{Q}_{z} \Gamma+\Lambda^{\prime} \mathcal{S} \Lambda \\
g & =\Gamma^{\prime} \mathcal{Q}_{z}(c-R)-\Lambda^{\prime} \mathcal{S} I_{0} u_{-1} \\
\rho & =\frac{1}{2}\|c-R\|_{\mathcal{Q}_{z}}^{2}+\frac{1}{2}\left\|u_{-1}\right\|_{S}^{2}
\end{aligned}
$$

Consequently, we may solve the FIR based MPC regulator problem (5) by solution of the following convex quadratic program

$$
\begin{array}{ll}
\min _{U} & \psi=\frac{1}{2} U^{\prime} H U+g^{\prime} U \\
\text { s.t. } & U_{\min } \leq U \leq U_{\max } \\
& b_{l} \leq \Lambda U \leq b_{u}
\end{array}
$$

in which

$$
U_{\min }=\left[\begin{array}{c}
u_{\min } \\
u_{\min } \\
\vdots \\
u_{\min }
\end{array}\right] \quad U_{\max }=\left[\begin{array}{c}
u_{\max } \\
u_{\max } \\
\vdots \\
u_{\max }
\end{array}\right]
$$

and

$$
b_{l}=\left[\begin{array}{c}
\Delta u_{\min }+u_{-1} \\
\Delta u_{\min } \\
\vdots \\
\Delta u_{\min }
\end{array}\right] \quad b_{u}=\left[\begin{array}{c}
\Delta u_{\max }+u_{-1} \\
\Delta u_{\max } \\
\vdots \\
\Delta u_{\max }
\end{array}\right]
$$

In a model predictive controller only the first vector, $u_{0}^{*}$, of $U^{*}=\left[\begin{array}{llll}\left(u_{0}^{*}\right)^{\prime} & \left(u_{1}^{*}\right)^{\prime} & \ldots & \left(u_{N-1}^{*}\right)^{\prime}\end{array}\right]^{\prime}$, is implemented on the process. At the next sample time the open-loop optimization is repeated with new information due to a new measurement. 


\section{Simple Estimator}

To have offset free steady state control when unknown step responses occur, we must have integrators in the feedback loop. This may be achieved using a FIR model in difference variables. Assume that the relation between the inputs and outputs may be represented as

$$
\Delta y_{k}=\Delta z_{k}=e_{k}+\sum_{i=1}^{n} H_{i} \Delta u_{k-i}
$$

in which $\Delta$ is the backward difference operator, i.e. $\Delta y_{k}=$ $y_{k}-y_{k-1}, \Delta z_{k}=z_{k}-z_{k-1}$, and $\Delta u_{k}=u_{k}-u_{k-1}$. This representation is identical with the FIR model (4)

$$
y_{k}=z_{k}=\hat{b}_{k}+\sum_{i=1}^{n} H_{i} u_{k-i}
$$

if $\hat{b}_{k}$ is computed by

$$
\begin{aligned}
& e_{k}=\Delta y_{k}-\sum_{i=1}^{n} H_{i} \Delta u_{k-i} \\
& \hat{b}_{k}=\hat{b}_{k-1}+e_{k}
\end{aligned}
$$

Note that in the regulator optimization problem $b_{1}=b_{2}=$ $\ldots=b_{N}=\hat{b}_{k}$ at each time instant. This is based on the assumption that the disturbances enter the process as constant output disturbances. Of course this may not be how the disturbances enter the process in practice, and significant performance deterioration may result as a consequence of this representation.

\section{UnCERTAin System Simulation}

In this Section we consider plants of the form

$$
Z(s)=Y(s)=G(s) U(s)+G_{d}(s) D(s)
$$

with the transfer functions

$$
\begin{aligned}
G(s) & =\frac{K(\beta s+1)}{\left(\tau_{1} s+1\right)\left(\tau_{2} s+1\right)} e^{-\tau s} \\
G_{d}(s) & =\frac{K_{d}\left(\beta_{d} s+1\right)}{\left(\tau_{d 1} s+1\right)\left(\tau_{d 2} s+1\right)} e^{-\tau_{d} s}
\end{aligned}
$$

The disturbance model, $G_{d}(s)$, is kept fixed at its nominal value, while the transfer function, $G(s)$, from $U(s)$ varies around its nominal value. This is used to illustrate the consequence of model uncertainty on the MPC closed-loop performance. The nominal system is $K=K_{d}=1, \tau_{1}=$ $\tau_{2}=\tau_{d 1}=\tau_{d 2}=5, \beta=\beta_{d}=2$, and $\tau=\tau_{d}=5$. The system is converted to discrete time using a sample time of $T_{s}=1$ and a zero-order-hold assumption on the inputs.

The predictive controller is based on the impulse response coefficients of the following system

$$
\hat{Z}(s)=\hat{Y}(s)=\hat{G}(s) U(s)
$$

in which $\hat{G}(s)$ is equal to the nominal system of $G(s)$. The simple estimator described in Section II-C is used for bias estimation. The input limits are $u_{\min }=-1, u_{\max }=1$, $\Delta u_{\min }=-0.2$, and $\Delta u_{\max }=0.2$. The horizon of the

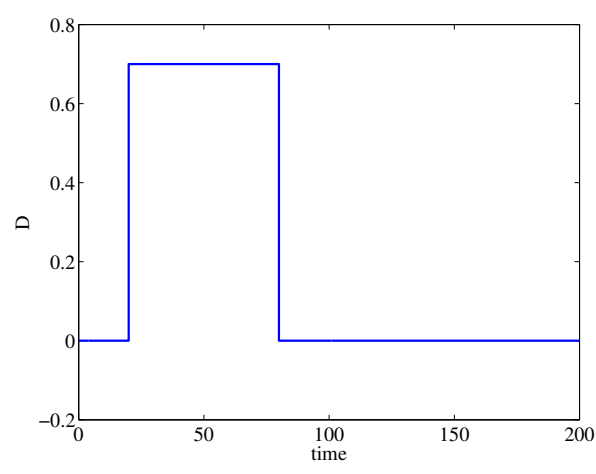

Fig. 2. Disturbance scenario.

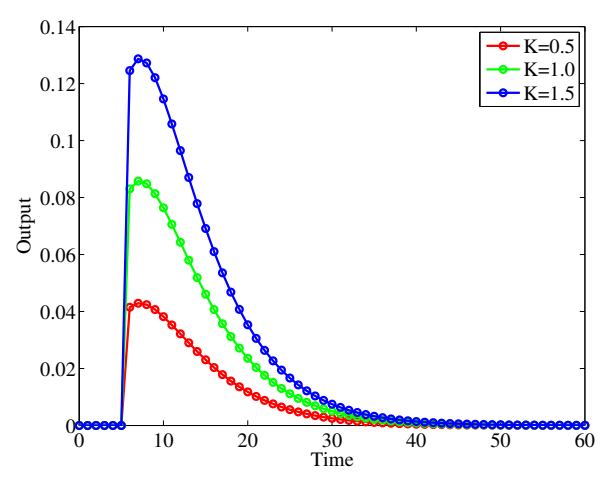

Fig. 3. Impulse responses for different gains, $K$, in (21a).

impulse response model is $n=40$ and the control horizon is $N=120$. The MPC is tuned with $Q_{z}=1$ and $S=10^{-3}$.

The performance of the controller on the different plants, $G(s)$, is evaluated using the disturbance function in Figure 2. This is an unmeasured disturbance and it is unknown to the controller.

\section{A. Effect of Uncertain Gain}

First we consider the effect of uncertainty in the gain, $K$. The impulse responses are illustrated in Figure 3. The corresponding closed-loop performance of the MPC is illustrated in Figure 4.

In Figure 3, it is evident that the gain uncertainty affect the impulse responses significantly. Consequently, there is a significant performance degradation of the closed-loop MPC response. This is evident in Figure 4. The steady state offset for the case $K=0.5$ in the period $50-100$ is due to an infeasible set point. The MV rides its lower limit and is too small (in an absolute sense) to reject the disturbance.

\section{B. Effect of Uncertain Time Constant}

Next, consider uncertainty in the time constant, $\tau_{1}$, of the system (21a). The impulse responses for different time constants, $\tau_{1}$, are illustrated in Figure 5. The corresponding closed-loop performance of the MPC is illustrated in Figure 6.

The effect of variations in the time constant, $\tau_{1}$, on the impulse responses is relatively small. Accordingly, the 

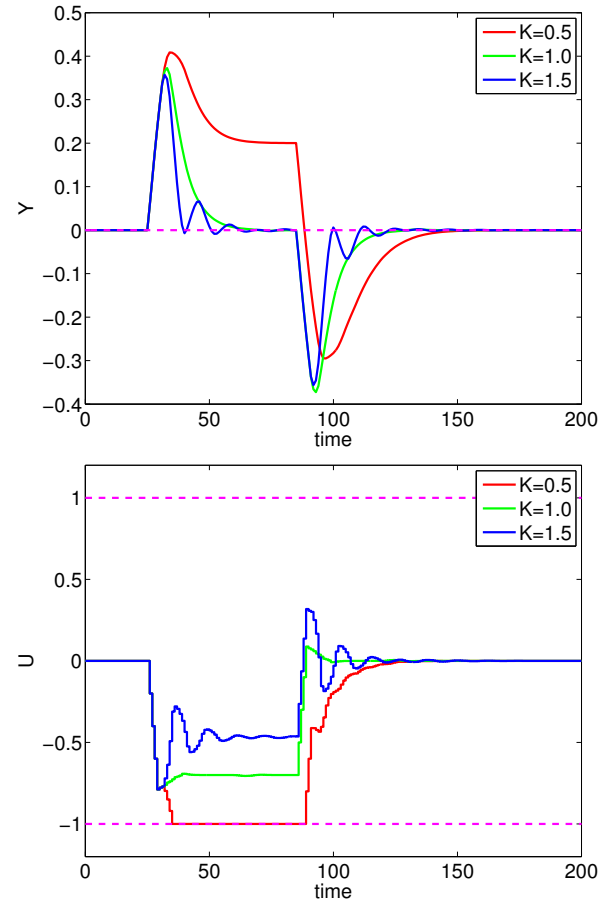

Fig. 4. Closed-loop MPC performance with gain uncertainty.

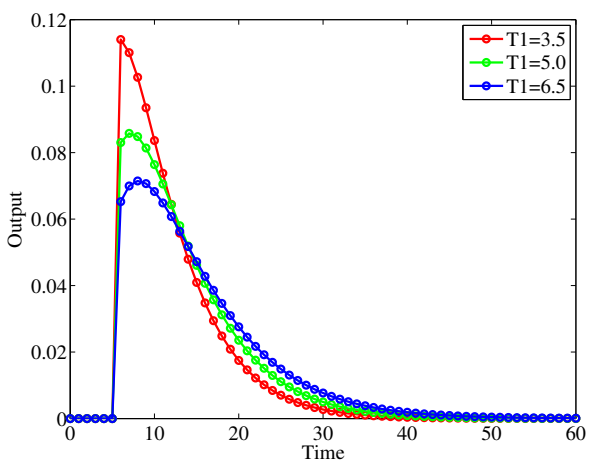

Fig. 5. Impulse responses for different time constants, $\tau_{1}$, in (21a).

degradation in the closed-loop MPC performance due to variations in the time constant, $\tau_{1}$, is modest.

\section{Effect of Uncertain $\beta$}

The impulse responses for different values of $\beta$ are illustrated in Figure 7. This is related to an uncertain zero of the system. It is evident that the impulse response with $\beta=4$ is much more different from the nominal impulse response $(\beta=2)$ than the impulse response with $\beta=0$. As is evident from Figure 8, the performance degradation for the case $\beta=$ 4 is also more pronounced than the performance degradation for the case $\beta=0$. This confirms that the uncertainty of the impulse response coefficients are well suited to measure the resulting closed-loop MPC performance degradation.

\section{Effect of Uncertain Time Delay}

The effect of variations in the time delay, $\tau$, of (21a) on the impulse responses is illustrated in Figure 9. The
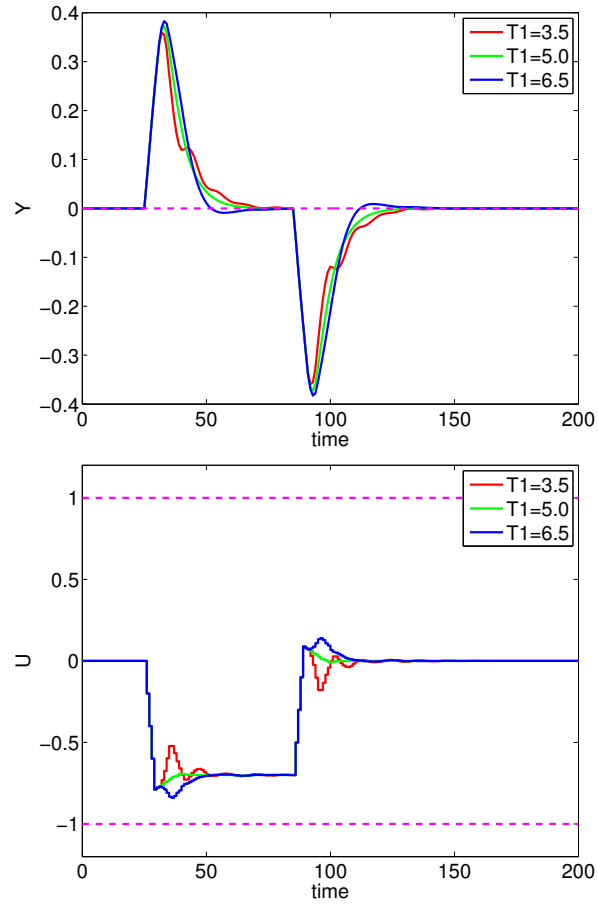

Fig. 6. Closed-Loop performance with time constant, $\tau_{1}$, variations.

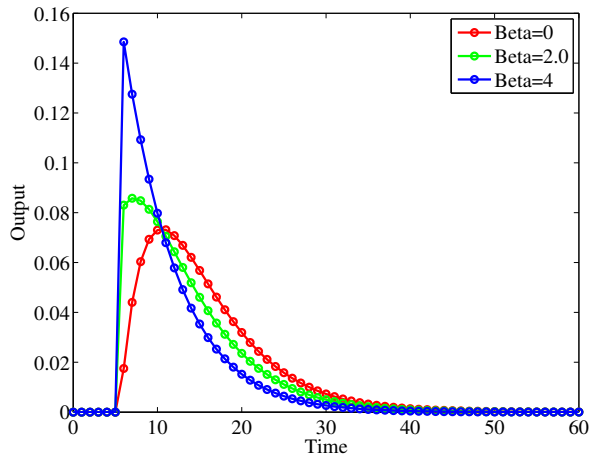

Fig. 7. Impulse responses for different values of $\beta$ in (21a).

responses are only slightly shifted horizontally. However, the vertical differences from the response of the nominal delay to the responses of the perturbed delays are large. Accordingly, this situation with variations in the time delay corresponds to significant impulse response uncertainty. As a consequence of the significant impulse response uncertainty, the degradation of the closed-loop MPC performance is significant as illustrated in Figure 10.

\section{Stochastic System Simulation}

The effect of process noise and measurement noise as well as model uncertainty on the closed-loop MPC performance is investigated in this section. We assume that the process noise enters the system in the same way as the unmeasured disturbance, i.e. $G=B_{d}$. The simulations in this section are based on the process noise and measurement noise illustrated in Figure 11. The signals are generated using a process noise variance of $Q=0.01^{2}$ and a measurement noise variance of 

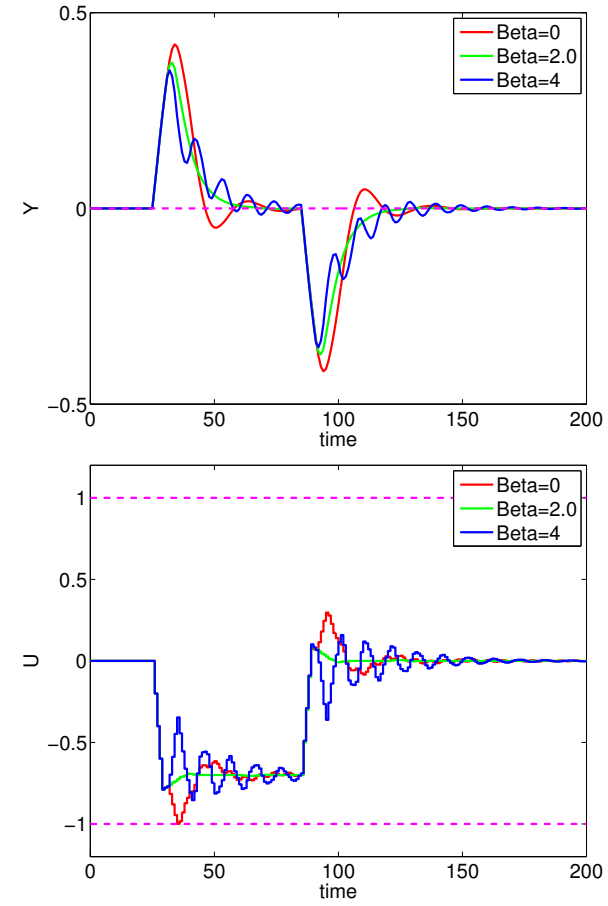

Fig. 8. Closed-loop MPC response for uncertain values of $\beta$ in the plant (21a).

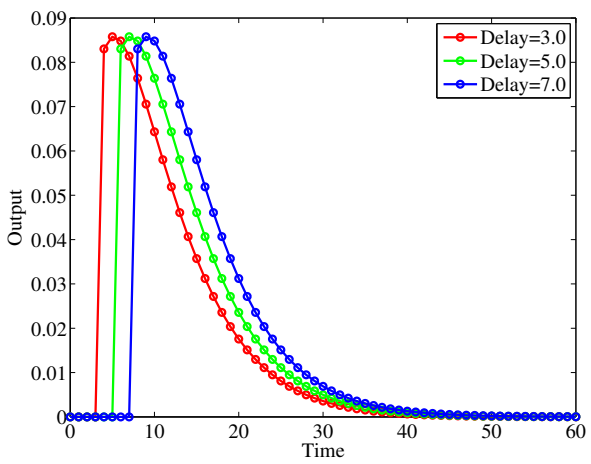

Fig. 9. Impulse responses for different time delays, $\tau$, in (21a).

$R=0.005^{2}$. The steady state $\left(x_{0}=0\right)$ is used as the initial state for the simulations.

The closed-loop MPC performance in the case when the process model equals the nominal model used for controller design is illustrated in Figure 12. Obviously, the performance is degraded compared to the deterministic case. As is evident in Figure 13, the closed-loop performance degrades further and becomes quite oscillatory in the case when there is a gain mismatch $(K=1.5)$. The effect of a time constant mismatch $\left(\tau_{1}=6.5\right)$ is illustrated in Figure 14. In this case the performance degradation compared to the nominal case is less pronounced. As illustrated in Figures 15 and 16, the closed-loop response degrades significantly and becomes quite oscillatory in the case of a zero mismatch $(\beta=4)$ and a time delay mismatch $(\tau=7)$. For this realization of the stochastic signals the effect of a zero mismatch seems to lead to the largest performance degradation.
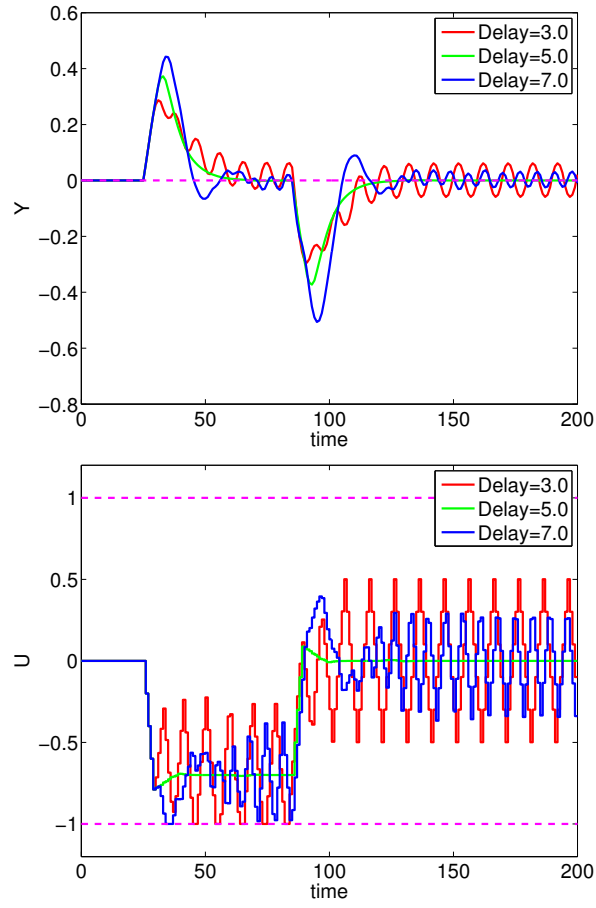

Fig. 10. Closed-loop MPC performance for different plant time delays.
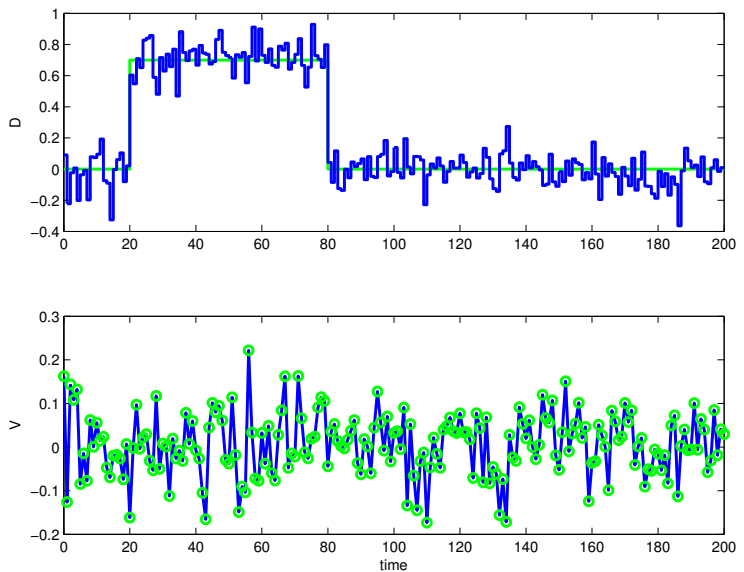

Fig. 11. Top: Deterministic disturbance function with added process noise. Bottom: Measurement noise.

\section{CONCLUSION}

Based on finite impulse response predictions, we have developed a regularized $l_{2}$ predictive controller with input and input-rate constraints. It is verified by simulations that the closed-loop MPC performance degradation due to plantmodel mismatch is tightly related to the uncertainty of the impulse response coefficients. The affine nature of the FIR model implies that it can be directly applied in predictive controllers based on robust linear programming [2] as well as predictive controllers based on second-order cone programming [3], [4]. The simulations in the present paper illustrate the potential as well as expected limits on the performance improvement that can be achieved by robust MPC, i.e. an upper limit on the potential performance is the performance 

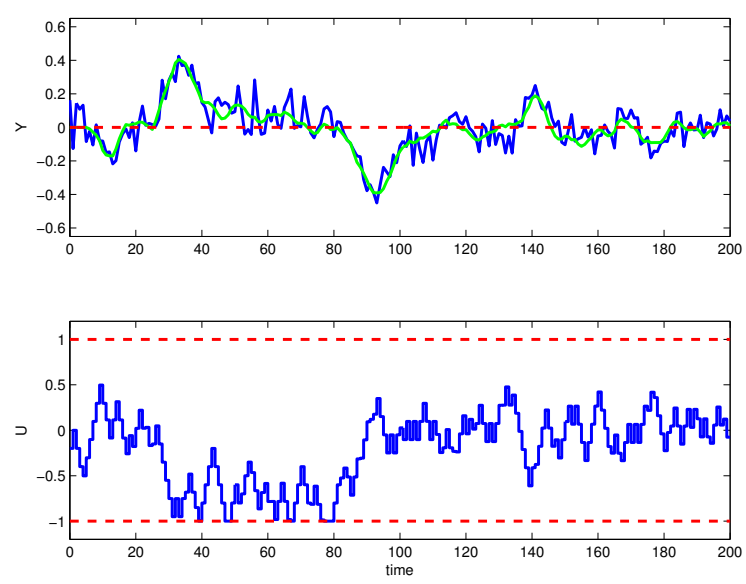

Fig. 12. Closed-loop MPC performance for the nominal system.
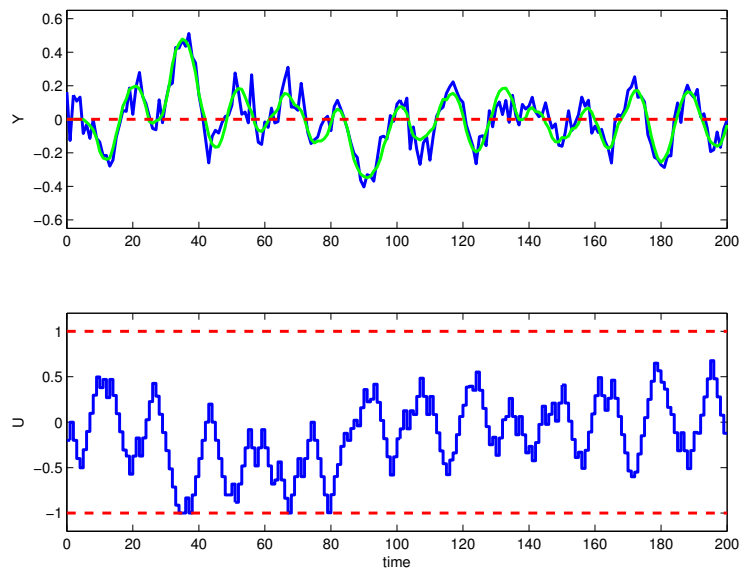

Fig. 13. Closed-loop MPC performance for the case $K=1.5$.

of the nominal model. The closed-loop performance can also be improved by adopting a FIR based moving horizon estimator instead of the simple estimator used in this paper.

\section{REFERENCES}

[1] J. M. Maciejowski, Predictive Control with Constraints. Harlow, England: Prentice Hall, 2002.

[2] L. Vandenberghe, S. Boyd, and M. Nouralishadi, "Robust linear programming and optimal control," in 15th IFAC World Congress. Barcelona: IFAC, 2002.

[3] A. Ben-Tal and A. Nemirovski, "Lectures on modern convex optimization," Philadelphia, 2001.

[4] S. Boyd and L. Vandenberghe, Convex Optimization. Cambridge, UK: Cambridge University Press, 2004.
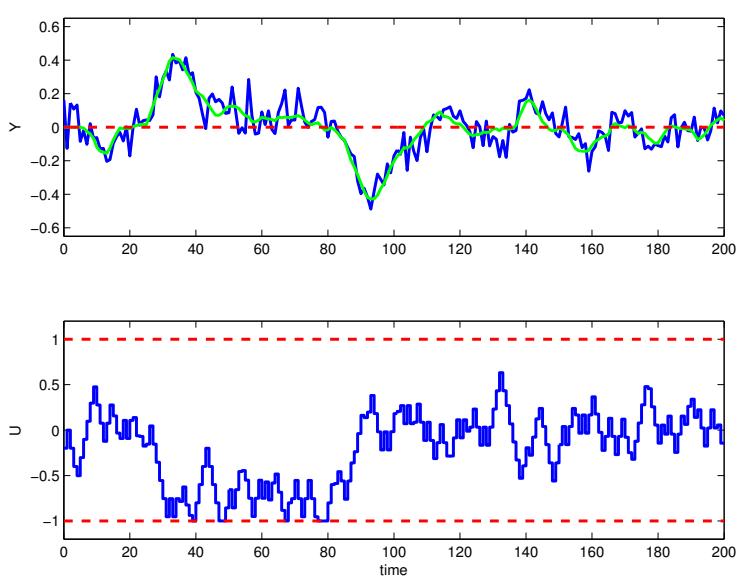

Fig. 14. Closed-loop MPC performance for the case $\tau_{1}=6.5$.
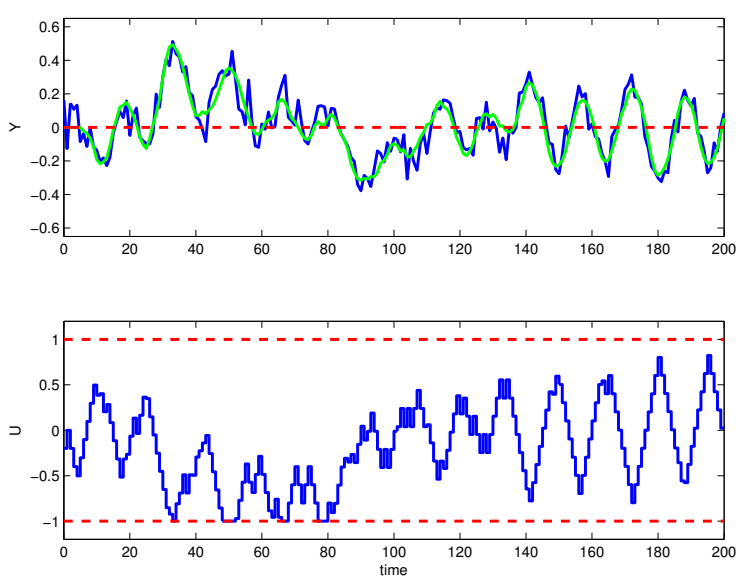

Fig. 15. Closed-loop MPC performance for the case $\beta=4$.
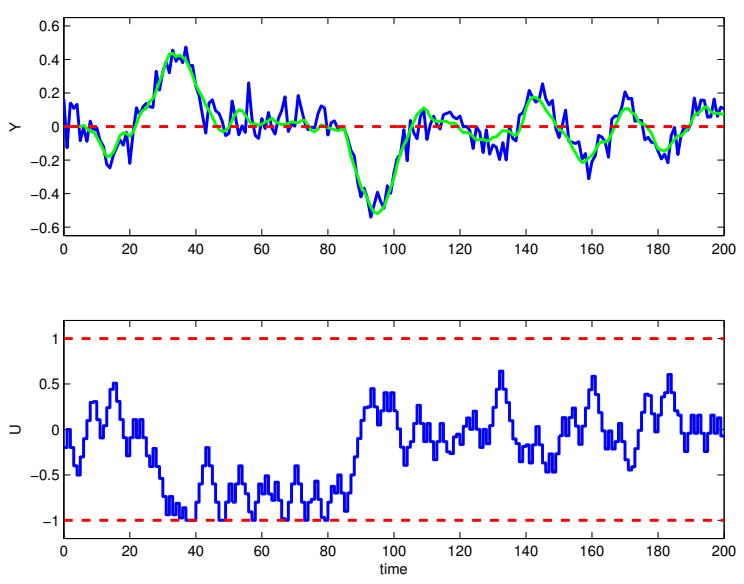

Fig. 16. Closed-loop MPC performance for the case $\tau=7$. 